TRANSACTIONS OF THE AMERICAN MATHEMATICAL SOCIETY

Volume 348, Number 10, October 1996

\title{
COMPETITIVE EXCLUSION AND COEXISTENCE FOR COMPETITIVE SYSTEMS ON ORDERED BANACH SPACES
}

\author{
S. B. HSU, H. L. SMITH, AND PAUL WALTMAN
}

\begin{abstract}
The dynamics of competitive maps and semiflows defined on the product of two cones in respective Banach spaces is studied. It is shown that exactly one of three outcomes is possible for two viable competitors. Either one or the other population becomes extinct while the surviving population approaches a steady state, or there exists a positive steady state representing the coexistence of both populations.
\end{abstract}

\section{INTRODUCTION}

The amount of research devoted to mathematical models of two competing populations is enormous. Most such models consist of ordinary differential equations or difference equations, but more recently models consisting of delay differential equations, partial differential equations, and even partial differential equations with delays have been studied. As the list of papers on the subject is very large, we content ourselves by referencing the works [6]-[10], [15], [16], [18]. Obviously, this is a biased list of references on the subject. Despite the fact that there are techniques of analysis that are common to a large body of this research, it was not until very recently that an abstract approach to competition was taken. The work of Hess and Lazer [7] seems to be the first such work. Another paper on the subject is Hsu, Waltman and Ellermeyer [9].

In the present paper, we continue the study of abstract competitive systems but in contrast to the approach in [7], it is not assumed that the competitive system is continuously differentiable.

The basic setup is as follows. For $i=1,2$, let $X_{i}$ be ordered Banach spaces with positive cones $X_{i}^{+}$such that $\operatorname{Int} X_{i} \neq \emptyset$. We use the same symbol for the partial orders generated by the cones $X_{i}^{+}$. If $x_{i}, \bar{x}_{i} \in X_{i}$, then we write $x_{i} \leq \bar{x}_{i}$ if $\bar{x}_{i}-x_{i} \in X_{i}^{+}, x_{i}<\bar{x}_{i}$ if $x_{i} \leq \bar{x}_{i}$ and $x_{i} \neq \bar{x}_{i}$, and $x_{i} \ll \bar{x}_{i}$ if $\bar{x}_{i}-x_{i} \in \operatorname{Int} X_{i}^{+}$. If $x_{i}, y_{i} \in X_{i}$ satisfy $x_{i}<y_{i}$, then the order interval $\left[x_{i}, y_{i}\right]$ is defined by $\left[x_{i}, y_{i}\right]=$

Received by the editors March 5, 1995

1991 Mathematics Subject Classification. Primary 47H07, 47H20; Secondary 92A15 .

Key words and phrases. Discrete order-preserving semigroup, order-preserving semiflow, positive fixed points, competitive systems, ejective fixed points.

Research of the first author was supported by the National Science Council, Republic of China. Research of the second author was supported by NSF Grant DMS 9300974.

Research of the third author was supported by NSF Grants DMS 9204490 and 9424592.

The third author wishes to express his thanks to Professor Peter Takáĉ for many stimulating discussions on ordered spaces and monotone operators. 
$\left\{u \in X_{i}: x_{i} \leq u \leq y_{i}\right\}$. If $x_{i} \ll y_{i}$ then $\left[\left[x_{i}, y_{i}\right]\right]=\left\{u \in X_{i}: x_{i} \ll u \ll y_{i}\right\}$ is called an open order interval.

Let $X=X_{1} \times X_{2}, X^{+}=X_{1}^{+} \times X_{2}^{+}$, and $K=X_{1}^{+} \times\left(-X_{2}^{+}\right) . X^{+}$is a cone in $X$ with nonempty interior given by $\operatorname{Int} X^{+}=\operatorname{Int} X_{1}^{+} \times \operatorname{Int} X_{2}^{+}$. It generates the order relations $\leq,<, \ll$ in the usual way. In particular, if $x=\left(x_{1}, x_{2}\right)$ and $\bar{x}=\left(\bar{x}_{1}, \bar{x}_{2}\right)$, then $x \leq \bar{x}$ if and only if $x_{i} \leq \bar{x}_{i}$, for $i=1,2$. For our purposes, the more important cone is $K$ which also has nonempty interior given by $\operatorname{Int} K=\operatorname{Int} X_{1}^{+} \times\left(-\operatorname{Int} X_{2}^{+}\right)$. It generates the partial order relations $\leq_{K},<_{K}, \ll_{K}$. In this case,

$$
x \leq_{K} \bar{x} \Longleftrightarrow x_{1} \leq \bar{x}_{1} \quad \text { and } \quad \bar{x}_{2} \leq x_{2} .
$$

A similar statement holds with $\ll_{K}$ replacing $\leq_{K}$ and $\ll$ replacing $\leq$.

Let $T: X^{+} \rightarrow X^{+}$be continuous and denote by $T^{n}$ the $n$-fold composition of $T$. The following hypotheses on $T$ are meant to capture the essence of competition between two adequate competitors (ones which can survive in the absence of competition).

(H1) $T$ is order compact and strictly order-preserving with respect to $<_{K}$. That is, $x<_{K} \bar{x}$ implies $T(x)<_{K} T(\bar{x})$.

(H2) $T(0)=0$ and 0 is a repelling fixed point. By repelling we mean there exists a neighborhood $U$ of 0 in $X^{+}$such that for each $x \in U, x \neq 0$, there is an integer $n=n(x)$ such that $T^{n}(x) \notin U$.

(H3) $T\left(X_{1}^{+} \times\{0\}\right) \subset X_{1}^{+} \times\{0\}$. There exists $\hat{x}_{1}$ satisfying $0 \ll \hat{x}_{1}$ such that $T\left(\left(\hat{x}_{1}, 0\right)\right)=\left(\hat{x}_{1}, 0\right)$, and $T^{n}\left(\left(x_{1}, 0\right)\right) \rightarrow\left(\hat{x}_{1}, 0\right)$ for every $x_{1}$ satisfying $0<x_{1}$. The symmetric conditions hold for $T$ on $\{0\} \times X_{2}$. The fixed point is denoted by $\left(0, \tilde{x}_{2}\right)$.

(H4) If $x, y \in X^{+}$satisfy $x<_{K} y$ and either $x$ or $y$ belongs to $\operatorname{Int} X^{+}$, then $T(x) \ll_{K}$ $T(y)$. If $x=\left(x_{1}, x_{2}\right) \in X^{+}$satisfies $x_{i} \neq 0, i=1,2$, then $T(x) \gg 0$.

Recall that $T$ is order compact if for every $\left(x_{1}, x_{2}\right) \in X^{+}$, it follows that $T\left(\left[0, x_{1}\right] \times\left[0, x_{2}\right]\right)$ has compact closure in $X$.

The strict order preserving property described in (H1) is the signature of a competitive system. It is biologically intuitive. The two related states, $x=\left(x_{1}, x_{2}\right)$ and $\bar{x}=\left(\bar{x}_{1}, \bar{x}_{2}\right)$, where $x<_{K} \bar{x}$, represent initial conditions in which the state of the first population is given by the first component and the state of the second population is given by the second component. The relation says that the second population has an advantage over the first in the state $x$ relative to the state $\bar{x}$ since the second population is greater in state $x$ and its competitors population is smaller. Viewed differently, population one has the advantage over population two in state $\bar{x}$. The order preservation property says merely that the relative advantage of one state over the other is preserved into the future.

We introduce the following notation for the "boundary" fixed points of $T$ :

$$
E_{0}=(0,0), \quad E_{1}=\left(\hat{x}_{1}, 0\right), \quad E_{2}=\left(0, \tilde{x}_{2}\right) .
$$

We say that a fixed point $E_{*}$ of $T$ is positive if it belongs to the interior of $X^{+}$. The order interval $I$ defined by

$$
I \equiv\left[0, \hat{x}_{1}\right] \times\left[0, \tilde{x}_{2}\right]
$$

will play an important role. 
Given $x \in X^{+}$we write $O(x)=\left\{T^{n}(x): n \geq 0\right\}$ for the positive orbit of $x$. Its omega limit set is defined in the usual way as

$$
\omega(x)=\left\{y \in X^{+}: T^{n_{i}}(x) \rightarrow y, \quad \text { some sequence }\left\{n_{i}\right\} \text { satisfying } n_{i} \rightarrow \infty\right\} .
$$

Our main result says that, for a competitive system, either there is a positive fixed point of $T$, representing coexistence of the two populations, or one population drives the other to extinction.

Theorem A. Let (H1)-(H4) hold. Then the omega limit set of every orbit is contained in I and exactly one of the following holds:

(a) There exists a positive fixed point $E_{*}$ of $T$ in $I$.

(b) $T^{n}(x) \rightarrow E_{1}$ as $n \rightarrow \infty$ for every $x=\left(x_{1}, x_{2}\right) \in I$ with $x_{i} \neq 0, i=1,2$.

(c) $T^{n}(x) \rightarrow E_{2}$ as $n \rightarrow \infty$ for every $x=\left(x_{1}, x_{2}\right) \in I$ with $x_{i} \neq 0, i=1,2$. Finally, if (b) or (c) holds and $x=\left(x_{1}, x_{2}\right) \in X^{+} \backslash I$ satisfies $x_{i} \neq 0, i=1,2$, then either $T^{n}(x) \rightarrow E_{1}$ or $T^{n}(x) \rightarrow E_{2}$.

In case (b) (and (c)) our result may seem a bit unsatisfactory in the sense that we do not conclude $T^{n}(x) \rightarrow E_{1}$ for all $x=\left(x_{1}, x_{2}\right)$ with $x_{i} \neq 0, i=1,2$. More precisely, we cannot rule out that there is such an $x \in X^{+} \backslash I$ such that $T^{n}(x) \rightarrow E_{2}$. In fact, we give an example of such behavior at the conclusion of this section. If such a point exists, then it is easy to see that $E_{2}$ attracts the set $\left\{y \in X^{+}: y \leq_{K} T(x)\right\}$ which has nonempty interior in $X^{+}$. As $E_{2}$ also repels a relatively open set in $I$ in case (b), $E_{2}$ would certainly be non-hyperbolic if $T$ were a smooth map.

If one is concerned only in establishing that exactly one of alternatives (a)-(c) of Theorem A holds and not at all in the behavior of $T$ outside of $I$, i.e., one is not interested in the first and last assertions of the theorem, then $(\mathrm{H} 1)-(\mathrm{H} 4)$ can be appropriately weakened so as to hold only in $I$. For example, the third sentence of (H3) would require convergence of the orbit of $\left(x_{1}, 0\right)$ to $\left(\hat{x}_{1}, 0\right)$ only for $0<x_{1}<\hat{x}_{1}$.

As a consequence of (H4) and the fact that the omega limit set of every orbit is contained in $I$, it follows that any fixed point, $E$, of $T$, distinct from the boundary fixed points (1.1), is necessarily a positive fixed point satisfying

$$
E_{2} \ll_{K} E \ll_{K} E_{1} .
$$

In [7] it is shown that if $T$ is $C^{1}$ and the spectral radius of its derivative at $E_{i}$ is smaller than one for $i=1,2$ or larger than one for $i=1,2$, then (a) holds. Our result is more general.

The following corollary of Theorem A and its proof provides sufficient conditions for the existence of a positive fixed point of $T$.

Corollary 1. Let (H1)-(H4) hold. Then $T$ has a positive fixed point if any one of the following holds.

(i) Both $E_{1}$ and $E_{2}$ are stable relative to I with respect to the order topology.

(ii) Both $E_{1}$ and $E_{2}$ are unstable relative to I with respect to the order topology.

(iii) There is a point $x \in X^{+}$and a point $z \in \omega(x)$ such that $z \gg 0$.

The order topology on $X$ is the topology generated by the open order intervals $\left[\left[x_{1}, y_{1}\right]\right] \times\left[\left[x_{2}, y_{2}\right]\right]$ where $x_{i} \ll y_{i}$. It is a norm topology (see [1]) and every order open set is open in the usual topology of $X$. A fixed point $x \in I$ is stable relative to $I$ in the order topology if for every order open set $U$ containing $x$ there is an order open set $V$ containing $x$ such that if $y \in V \cap I$, then $T^{n}(y) \in U \cap I$ for all $n \geq 1$. If this does not hold, we say that $x$ is unstable relative to $I$ in the order 
topology. If the cones $X_{i}^{+}$are normal (there exists $k_{i}>0$ such that $0 \leq x_{i} \leq y_{i}$ implies $\left.\left\|x_{i}\right\| \leq k_{i}\left\|y_{i}\right\|\right)$, then the order topology coincides with the usual topology (see [1]).

As a special case of (iii), if the dynamical system generated by $T$ is uniformly persistent (see [4]) then there is a positive fixed point. In fact, it suffices to assume that $z=\left(z_{1}, z_{2}\right)$ in (iii) satisfies $z_{i} \neq 0$ since then $T z \gg 0$, by (H4), and $T z$ belongs to $\omega(x)$. Consequently, if $T$ has a periodic point $z=\left(z_{1}, z_{2}\right)$ of period $p>1$ with $z_{i}>0, i=1,2$, then $T$ has a positive fixed point.

The existence of a positive fixed point has implications for the stability of $E_{1}$ and $E_{2}$ as the next result shows.

Proposition 2. Let (H1)-(H4) hold and suppose that $T$ has a positive fixed point. Suppose further that $E_{1}$ is an isolated fixed point of $T$. Then there exists a positive fixed point $E_{*}$ in I such that exactly one of the following holds:

(i) $T^{n}(x) \rightarrow E_{*}$ for all $x=\left(x_{1}, x_{2}\right)$ satisfying $E_{*} \leq_{K} x<_{K} E_{1}$ and $x_{2} \neq 0$.

(ii) $T^{n}(x) \rightarrow E_{1}$ for all $x$ satisfying $E_{*}<_{K} x \leq_{K} E_{1}$.

A symmetric conclusion holds if it is assumed that $E_{2}$ is an isolated fixed point.

The last assertion of Proposition 2 says that if $E_{2}$ is an isolated fixed point, then there exists a positive fixed point $E_{* *}$ satisfying (1.2) such that one of the following holds: (i) $T^{n}(x) \rightarrow E_{* *}$ for all $x=\left(x_{1}, x_{2}\right)$ satisfying $E_{2}<_{K} x \leq_{K} E_{* *}$ and $x_{1} \neq 0$, or (ii) $T^{n}(x) \rightarrow E_{2}$ for all $x$ satisfying $E_{2} \leq_{K} x<_{K} E_{* *}$. If both $E_{1}$ and $E_{2}$ are isolated fixed points, then it follows from the proof of Proposition 2 that $E_{2} \ll_{K} E_{* *} \leq_{K} E_{*} \ll_{K} E_{1}$ with equality $E_{* *}=E_{*}$ possible. However, if equality does not hold, then $E_{* *} \ll_{K} E_{*}$, by (H4). In that case, the proof of Theorem A implies that exactly one of three alternatives holds: (1) there is a fixed point $E$ satisfying $E_{* *} \ll_{K} E \ll_{K} E_{*}$, or $(2) T^{n}(x) \rightarrow E_{* *}$ for all $x$ satisfying $E_{* *} \leq_{K} x<_{K} E_{*}$, or (3) $T^{n}(x) \rightarrow E_{*}$ for all $x$ satisfying $E_{* *}<_{K} x \leq_{K} E_{*}$. It is clear that this reasoning can be extended further. In fact, by Zorn's Lemma, there exists a maximal totally ordered (by $<_{K}$ ) set of equilibria containing the minimal element $E_{2}$ and the maximal element $E_{1}$.

One of the motivations for the present study was the conjecture that the boundary of the basin of attraction of, say $E_{1}$, must contain a positive fixed point in the case that $E_{1}$ is a local attractor but not a global attractor. $E_{1}$ is a local attractor if $T^{n}(x) \rightarrow E_{1}$ for all $x$ in some relatively open subset of $X^{+}$containing $E_{1}$. Obviously, if $E_{1}$ is a local attractor but alternative (b) of Theorem A does not hold, then alternative (a) must hold and case (ii) of Proposition 2 must hold.

We now formulate a continuous-time version of Theorem A. Assume that $T$ : $[0, \infty) \times X^{+} \rightarrow X^{+}$is a continuous semiflow. We write $T_{t}(x)=T(t, x)$. The semiflow properties are (i) $T_{0}(x)=x$ for all $x \in X^{+}$, and (ii) $T_{t} \circ T_{s}=T_{t+s}$ for $t, s \geq 0$. The analogous hypotheses to (H1)-(H4) above are given below.

(H1) $T$ is strictly order-preserving with respect to $<_{K}$. That is, $x<_{K} \bar{x}$ implies $T_{t}(x)<_{K} T_{t}(\bar{x})$. For each $t>0, T_{t}: X^{+} \rightarrow X^{+}$is order compact.

(H2) $T_{t}(0)=0$ for all $t \geq 0$ and 0 is a repelling equilibrium. There exists a neighborhood $U$ of 0 in $X^{+}$such that for each $x \in U, x \neq 0$, there is a $t_{0}>0$ such that $T_{t_{0}}(x) \notin U$.

(H3) $T_{t}\left(X_{1}^{+} \times\{0\}\right) \subset X_{1}^{+} \times\{0\}$ for all $t \geq 0$. There exists $\hat{x}_{1} \gg 0$ such that $T_{t}\left(\left(\hat{x}_{1}, 0\right)\right)=\left(\hat{x}_{1}, 0\right)$ for all $t \geq 0$, and $T_{t}\left(\left(x_{1}, 0\right)\right) \rightarrow\left(\hat{x}_{1}, 0\right)$ as $t \rightarrow \infty$ for all 
$x_{1} \neq 0$. The symmetric conditions hold for $T$ on $\{0\} \times X_{2}^{+}$with equilibrium point $\left(0, \tilde{x}_{2}\right)$.

(H4) If $x=\left(x_{1}, x_{2}\right) \in X^{+}$satisfies $x_{i} \neq 0, i=1,2$, then $T_{t}(x) \gg 0$ for $t>0$. If $x, y \in X^{+}$satisfy $x<_{K} y$ and either $x$ or $y$ belongs to $\operatorname{Int} X^{+}$, then $T_{t}(x) \ll_{K}$ $T_{t}(y)$ for $t>0$.

As in the discrete case, the boundary equilibria are given by (1.1). We say that $E_{*}$ is a positive equilibrium of $T$ if it belongs to the interior of $X^{+}$. If $x \in X^{+}$ then $O(x)=\left\{T_{t}(x): t \geq 0\right\}$ is called the positive orbit of $T$. Its omega limit set is defined in the usual way.

Theorem B. Let (H1)-(H4) hold. Then the omega limit set of every orbit is contained in $I$ and exactly one of the following holds:

(a) There exists a positive equilibrium $E_{*}$ of $T$ in $I$.

(b) $T_{t}(x) \rightarrow E_{1}$ as $t \rightarrow \infty$ for every $x=\left(x_{1}, x_{2}\right) \in I$ with $x_{i} \neq 0, i=1,2$.

(c) $T_{t}(x) \rightarrow E_{2}$ as $t \rightarrow \infty$ for every $x=\left(x_{1}, x_{2}\right) \in I$ with $x_{i} \neq 0, i=1,2$.

Finally, if (b) or (c) hold, $x=\left(x_{1}, x_{2}\right) \in X^{+} \backslash I$ and $x_{i} \neq 0, i=1,2$, then either $T_{t}(x) \rightarrow E_{1}$ or $T_{t}(x) \rightarrow E_{2}$ as $t \rightarrow \infty$.

The obvious counterparts to Corollary 1 and Proposition 2 hold in the continuous case as well.

The following example shows that case (c) of Theorem B may hold yet some open set of initial data outside $I$ is attracted to $E_{1}$. Consider the planar system

$$
\begin{aligned}
& x_{1}^{\prime}=x_{1}\left(1-x_{1}-\mu x_{2}\right)^{3} \\
& x_{2}^{\prime}=x_{2}\left(1-x_{1}-x_{2}\right)
\end{aligned}
$$

where $\mu>1$. It is easy to verify that all positive solutions beginning in $I=$ $[0,1] \times[0,1]$ are attracted to $E_{2}=(0,1)$ but that solutions starting at $\left(x_{1}, x_{2}\right)$ near $E_{1}=(1,0)$ and satisfying $x_{1}>1,0<x_{2}<\left(x_{1}-1\right)^{2}$ are attracted to $E_{1}$. The time-one map corresponding to the flow gives a similar example for Theorem A.

The semiflow $T$ on $X^{+}$generates a monotone dynamical system which is strongly monotone on Int $X^{+}$but not on $X^{+}$. For strongly monotone systems, M.W. Hirsch [5] showed that the generic solution converges to the set of equilibria. This result was later improved by Polacik [14] and Smith and Thieme [17], under additional smoothness conditions, to conclude that the generic solution converges to a single equilibrium. See [16] for a self-contained treatment. In the present case, these results do not generally apply but see [10], [15], [16], [18] for cases in which they do.

The main tool in our proof of Theorem A is an extension of a result of Dancer and Hess [2] concerning a strictly order preserving map on an order interval generated by two ordered fixed points. This extension, treated in the next section, is of independent interest. The main results are proved in a subsequent section.

\section{The trichotomy of Dancer and Hess}

Let $Y$ be a Banach space and $Y^{+}$be a cone in $Y$. As usual, we denote by $\leq$ and $<$ the partial order relation generated by $Y^{+}$. If $Y^{+}$has nonempty interior in $Y$, we write $x \ll y$ if $y-x \in \operatorname{Int} Y^{+}$. If $x<y$, we define $[x, y]=\{u \in Y: x \leq u \leq y\}$ and refer to it as the order interval generated by $x$ and $y$. If $x \ll y$, set $[[x, y]]=$ $\{u \in Y: x \ll u \ll y\}$. If $C$ is a convex subset of $Y$ and $e \in C$, then $e$ is an extreme point of $C$ if there do not exist points $x, y \in C \backslash\{e\}$ such that $e=1 / 2(x+y)$. 
Let $U \subset Y$ and $S: U \rightarrow U$ be a continuous function. $S$ is strictly orderpreserving if

$$
x<y \Rightarrow S(x)<S(y) .
$$

A fixed point $u$ of $S$ is said to be an ejective fixed point if there is an open subset $V$ of $U$ containing $u$ such that for every $x \in V \backslash\{u\}$ there is an integer $m$ for which $S^{m}(x) \notin V$. In the previous section we called $u$ a repelling fixed point. However, the term ejective is more commonly used in the fixed point literature. Let $Z$ denote the set of integers. A sequence $\left\{x_{n}\right\}_{n \in Z}$ in $U$ is called an entire orbit of $S$ if $x_{n+1}=S\left(x_{n}\right)$ for all $n \in Z$. If there exist fixed points $u$ and $v$ of $S$ in $U$ such that $x_{n} \rightarrow v$ as $n \rightarrow \infty$ and $x_{n} \rightarrow u$ as $n \rightarrow-\infty$, then we say that the entire orbit joins $u$ to $v$.

The next result is a modification of a result of Dancer and Hess [2, Proposition 1]; see also Hess [6, Proposition 2.1].

Proposition 2.1. Let $u_{1}<u_{2}$ be fixed points of the strictly order-preserving continuous function $S: U \rightarrow U$, let $I \equiv\left[u_{1}, u_{2}\right] \subset U$, and suppose that $S(I)$ has compact closure in I. Suppose further that $S$ has an ejective fixed point $e \in I \backslash\left\{u_{1}, u_{2}\right\}$ which is an extreme point of I. Then at least one of the following holds:

(a) $S$ has a fixed point distinct from $u_{1}, u_{2}, e$ in $I$.

(b) There is an entire orbit $\left\{x_{n}\right\}_{n \in Z}$ of $S$ joining $u_{1}$ to $u_{2}$ and satisfying $x_{n+1}>$ $x_{n}, n \in Z$.

(c) There is an entire orbit $\left\{y_{n}\right\}_{n \in Z}$ of $S$ joining $u_{2}$ to $u_{1}$ and satisfying $y_{n+1}<$ $y_{n}, n \in Z$.

Proof. The proof is a minor modification of the proof given in [2] so we will merely indicate the modifications to that proof. The key point is that (Nussbaum [12], [13]) the fixed point index of an ejective fixed point that is an extreme point of $I$ vanishes, $i(S, I, e)=0$, and therefore from the assumption that (a) does not hold and the homotopy argument in [2] leading to $i\left(S, I, u_{2}\right)=1$ and $i(S, I, I)=1$, the additivity of the index still implies that $i\left(S, I, u_{1}\right)=0$ as in the proof in [2]. Furthermore, as $e$ is an ejective fixed point, no strictly monotone sequence of iterations $S^{n}(u)$ can satisfy $S^{n}(u) \rightarrow e$ as $n \rightarrow \infty$. As a technical point, one should also choose $\delta_{0}>0$ in the proof of Dancer and Hess so that $u_{2}, e \notin \bar{B}_{I}\left(0, \delta_{0}\right)$. The remainder of the proof in [2] is unaffected by the existence of $e$.

Our Proposition 2.1 differs from Proposition 1 in [2] by allowing for the existence of an ejective fixed point on the boundary of $I$ provided that it is an extreme point of $I$. It is clear from the proof that one can allow for any finite number of ejective fixed points that are also extreme points. Furthermore, as in Wu et al. [20] and noting the remarks following the proof of Theorem 3.4 in [13], the hypothesis that $S(I)$ has compact closure in $I$ can be relaxed to requiring that $S(I) \subset I$ and that $S$ is a strict-set-contraction with respect to a measure of noncompactness. Finally, the assumption that the ejective fixed point $e$ is an extreme point can be dropped if $I$ is not contained in any finite dimensional affine linear subspace of $Y$ and can be weakened in any event (see [12], [13]). Actually, we use only two properties of the fixed point $e$ implied by the assumptions that it is ejective and an extreme point. Namely, that its fixed point index is zero and that it is not the limit of a strictly monotone sequence of iterations. 
An ejective fixed point occurs naturally in competitive systems as formulated in section 1. The set $I$ can be viewed as an order interval $I=\left\{x \in X: E_{2} \leq_{K} x \leq_{K}\right.$ $\left.E_{1}\right\}$ containing the ejective fixed point $E_{0}$ which is an extreme point.

It is worth noting that as $u_{1}<e<u_{2}$, Proposition 1 of [2] applies to $S$ on $\left[u_{1}, e\right]$ and on $\left[e, u_{2}\right]$. Obviously, the corresponding alternative (b) of that result cannot hold for $S$ on $\left[u_{1}, e\right]$ since $e$ is an ejective fixed point. Similarly, the corresponding alternative (c) of that result cannot hold for $S$ on $\left[e, u_{2}\right]$.

The following example shows that more than one of the alternatives (a)-(c) may hold. Let $Y=\mathbb{R}^{2}$ with cone $Y^{+}=\left\{y \in Y: y_{i} \geq 0\right\}, \hat{0}=(0,0), \hat{1}=(1,1), I=[\hat{0}, \hat{1}]$, and let $S$ be the time-one map corresponding to the flow of the planar system:

$$
x^{\prime}=x(1-x), \quad y^{\prime}=y(1-y) .
$$

Clearly, $S$ is strictly order-preserving and, in addition to the fixed points $\hat{0}$ and $\hat{1}$, it fixes the points $(0,1)$ and $(1,0)$ and has a monotone entire orbit, $S^{n}(1 / 2,1 / 2), n \in$ $Z$, joining $\hat{0}$ to $\hat{1}$. Thus, alternatives (a) and (b) hold. It is easy to argue that if $Y^{+}$ has nonempty interior and $u_{1} \ll u_{2}$, then (b) and (c) cannot simultaneously hold. For in that case, since $y_{n} \rightarrow u_{2}\left(x_{n} \rightarrow u_{1}\right)$ as $n \rightarrow-\infty$, there exists an $n_{0} \in Z$ such that $u_{1} \ll y_{n_{0}}$ and there exists an $m_{0}$ such that $x_{m_{0}} \ll y_{n_{0}}$. As $S$ is strictly monotone, we get

$$
x_{m_{0}+l}=S^{l}\left(x_{m_{0}}\right)<S^{l}\left(y_{n_{0}}\right)=y_{n_{0}+l}, \quad l \geq 0 .
$$

Letting $l \rightarrow \infty$ leads to the contradiction $u_{2} \leq u_{1}$.

The next result says that if $Y^{+}$has nonempty interior in $Y$ and if $u_{1}, u_{2}, e$ are the only fixed points on the boundary of $I$, then precisely one of the alternatives (a)-(c) can hold.

Proposition 2.2. In addition to the hypotheses of Proposition 2.1, assume that $Y^{+}$ has nonempty interior in $Y$ and $u_{1} \ll u_{2}$. If $u \in I \backslash\left\{u_{1}, u_{2}, e\right\}$ and $S u=u$ implies that $u \in\left[\left[u_{1}, u_{2}\right]\right]$, then precisely one of the alternatives (a)-(c) of Proposition 2.1 holds.

Proof. We have already noted that $u_{1} \ll u_{2}$ implies that alternatives (b) and (c) are incompatible so it remains only to show that (a) and (b) and (a) and (c) are incompatible. Suppose that (a) and (b) hold for $S$ and let $\left\{x_{n}\right\}_{n \in Z}$ be the entire orbit described in (b). Then $S$ has a fixed point $u \in\left[\left[u_{1}, u_{2}\right]\right]$ and $x_{n_{0}} \ll u$ for some $n_{0}$ so we conclude from strict monotonicity of $S$ that $x_{n_{0}+l}<u$ for all $l \geq 0$. Letting $l \rightarrow \infty$ leads to the contradiction $u_{2} \leq u$. A similar contradiction follows in case (a) and (c) hold.

The following result is well-known but we include a proof for completeness. Recall that $x \in E$ is a maximal element of a set $E$ if $e \in E$ and $x \leq e$ implies that $x=e$. A minimal element is similarly defined.

Lemma 2.3. Let $E$ be a compact subset of $Y$. Then $E$ contains a maximal (minimal) element.

Proof. By Zorn's lemma, it suffices to show that if $F$ is a totally ordered subset of $E$, then $F$ has an upper bound in $E$. As $F$ can be viewed as a net in the compact space $E$, it has a cluster point $x \in E$. This means that given a neighborhood $U$ of $x$ and $f \in F$, there exists $f^{\prime} \in F$ satisfying $f \leq f^{\prime}$ and $f^{\prime} \in U$. Therefore, given $f \in F$ we can construct a sequence $f_{n}$ of points of $F$ such that $f \leq f_{n} \leq f_{n+1}$ 
for all $n$ and $f_{n} \rightarrow x$. Letting $n \rightarrow \infty$ in this inequality leads to $f \leq x$, and since $f \in F$ was arbitrary, $x$ is the desired upper bound.

We conclude this section by formulating a semiflow version of Proposition 2.1. An entire orbit of a semiflow $S=\left\{S_{t}\right\}_{t \geq 0}$ on $U$ is a continuous map $\gamma: \mathbb{R} \rightarrow U$ satisfying $S_{t}(\gamma(s))=\gamma(t+s)$ for $t \geq 0$ and $s \in \mathbb{R}$.

Proposition 2.4. Let $u_{1}<u_{2}$ be equilibria of the strictly order-preserving semiflow $S_{t}: U \rightarrow U$, let $I \equiv\left[u_{1}, u_{2}\right] \subset U$, and suppose that $S_{t}(I)$ has compact closure in $I$ for each $t>0$. Suppose further that $S$ has an ejective equilibrium $e \in I \backslash\left\{u_{1}, u_{2}\right\}$ which is an extreme point of I. Then at least one of the following holds:

(a) There is an equilibrium in I distinct from $u_{1}, u_{2}, e$.

(b) There is an entire orbit $\gamma: \mathbb{R} \rightarrow I$ satisfying $\gamma\left(t_{1}\right)<\gamma\left(t_{2}\right)$ if $t_{1}<t_{2}$ and $\gamma(-\infty)=u_{1}, \gamma(+\infty)=u_{2}$.

(c) There is an entire orbit $\gamma: \mathbb{R} \rightarrow I$ satisfying $\gamma\left(t_{2}\right)<\gamma\left(t_{1}\right)$ if $t_{1}<t_{2}$ and $\gamma(+\infty)=u_{1}, \gamma(-\infty)=u_{2}$.

Proof sketch. Our proof outline borrows key ideas from the outline given in [2], [6] but appears to be significantly different and less economical. As in [2], [6], we assume hereafter that alternative (a) does not hold. The following points address issues related to the ejective equilibrium $e$.

First, we verify that $e$ is an ejective fixed point of the map $S_{1 / n}$ for all large integers $n$. If $x \in Y$ and $r>0$, we denote by $B(r, x)$ the open ball of radius $r$ centered at $x$. As $e$ is an ejective equilibrium for the semiflow $S$, there exists $r>0$ such that for each $x \in \overline{B(r, e)} \cap I, x \neq e$, there is an $\epsilon=\epsilon(x)>0$ and $t=t(x)>0$ such that $S_{s}(y) \notin \bar{B}(r, e)$ for all $y \in B(\epsilon, x)$ and $s \in(t-\epsilon, t+\epsilon)$. As $C=\overline{S_{1}(\overline{B(r, e)} \cap I)} \cap \overline{B(r, e)}$ is compact, there is a finite subcover $\left\{B\left(\epsilon\left(x_{i}\right), x_{i}\right)\right\}_{i=1}^{p}$ of the cover $\{B(\epsilon(x), x)\}_{x \in C}$ of $C$. Consequently, if $x \in C$, then for some $i, S_{s}(x) \notin$ $\bar{B}(r, e)$ for $s \in\left(t_{i}-\epsilon_{i}, t_{i}+\epsilon_{i}\right)$, where $t_{i}=t\left(x_{i}\right)$ and $\epsilon_{i}=\epsilon\left(x_{i}\right)$. Now choose $N$ such that $N^{-1}<\epsilon_{i}, 1 \leq i \leq p$. We claim that $e$ is an ejective fixed point of the map $S_{1 / n}$ if $n \geq N$. We will show that if $x \in(B(r, e) \cap I) \backslash\{e\}$, then $S_{1 / n}^{k}(x) \notin B(r, e)$ for some positive integer $k$. If $S_{1}(x) \notin B(r, e)$, then we are done so we can suppose that $S_{1}(x) \in B(r, e)$ and therefore it belongs to $C$. Consequently, $S_{1+s}(x) \notin B(r, e)$ for all $s \in\left(t_{i}-\epsilon_{i}, t_{i}+\epsilon_{i}\right)$ for some $i=1,2, \ldots, p$. As $1 / n<\epsilon_{i}$, there is a positive integer $k$ such that $S_{1+k / n}(x) \notin B(r, e)$. This completes the proof of the claim.

Now we show that given $\epsilon>0$, there exists $\delta>0$ such that if $S_{t}(x)=x$ for some $x \in I$ and $t \in(0, \delta)$, then either $x=e$ or $x \in B\left(\epsilon, u_{1}\right) \cup B\left(\epsilon, u_{2}\right)$. First note that $J=\omega(I) \equiv \bigcap_{\tau>0} \overline{\bigcup_{t>\tau} S_{t}(I)}$ is nonempty, compact, invariant and attracts $I$ (see [3]). It is easy to see that if $S_{t}(x)=x$ for some $t>0$, then $x \in J$. If the claim is false, then for some $\epsilon>0$ and every integer $n \geq 1$, there are points $x_{n}$ and times $t_{n}>0$ with $t_{n} \rightarrow 0$ such that $S_{t_{n}}\left(x_{n}\right)=x_{n}, x_{n} \neq e$ and $\left\|x_{n}-u_{i}\right\| \geq \epsilon$. As $x_{n} \in J$, we can assume that $x_{n} \rightarrow x$ where $x \in I$. A standard argument establishes that $x$ is an equilibrium of the semiflow $S$. By assumption, the only equilibria are $u_{1}, u_{2}$ and $e$. Since $\left\|x-u_{i}\right\| \geq \epsilon$, it follows that $x \neq u_{i}, i=1,2$. But $x \neq e$ either as otherwise $x_{n} \in B(r, e) \backslash\{e\}$ for all large $n$, where $B(r, e)$ is as in the previous paragragh. By continuity of $S$, we may assume that $S_{t}\left(x_{n}\right) \in B(r, e)$ for $0 \leq t \leq 1$ for all large $n$. In that case, $S_{t}\left(x_{n}\right) \in B(r, e)$ for all $t \geq 0$, contradicting the ejectivity of $e$. This contradiction shows that $x \neq e$. As there are no other equilibria in $I$, we have reached a contradiction to our assumption that the claim is false. 
Using the results of the previous paragraph, for each positive integer $n$, we can choose a positive integer $m_{n}$ such that $m_{n}<1 / n$ and if $S_{1 / m_{n}}(x)=x$, then $x=e$ or $x \in B\left(1 / n, u_{1}\right) \cup B\left(1 / n, u_{2}\right)$. We can assume that $m_{n}<m_{n+1}$ for all $n$. Now applying Lemma 2.3 and the compactness of the set of fixed points, there exist a maximal fixed point $v_{n}$ of $S_{1 / m_{n}}$ in $B\left(1 / n, u_{1}\right)$ and a minimal fixed point $w_{n}$ in $B\left(1 / n, u_{2}\right) \cap\left[v_{n}, u_{2}\right]$. Consequently, the only fixed points of $S_{1 / m_{n}}$ in $\left[v_{n}, w_{n}\right]$ are $v_{n}, u_{n}$ and possibly $e$, if $v_{n}<e<w_{n}$. If the latter holds then $e$ is an extreme point of $\left[v_{n}, w_{n}\right]$ and an ejective fixed point. Now apply Proposition 2.1, if $e \in\left[v_{n}, w_{n}\right]$, or Proposition 1 of [2] otherwise, to conclude that for each $n$ there is an entire orbit $\left\{x_{k}^{n}\right\}_{k \in Z}$ of $S_{1 / m_{n}}$ satisfying either (1) $x_{k}^{n}<x_{k+1}^{n}$ and the orbit connects $v_{n}$ to $w_{n}$, or (2) $x_{k+1}^{n}<x_{k}^{n}$ and the orbit connects $w_{n}$ to $v_{n}$. By passing to a subsequence if necessary, we assume that (1) holds for all $n$. Note that for all $n,\left\{x_{k}^{n}\right\}_{k \in Z} \subset J$.

Let $F=\left\{x \in J: S_{t}(x) \geq x, t \geq 0\right\}$ be the set of subequilibria in $J$. Then $F$ is compact and positively invariant. Its omega limit set, $\omega(F)$, is invariant and contains $F$ (see [3]). It is easy to check that if, for each integer $l$, there exist a positive integer $n_{l}$ and an integer $k_{l}$ such that $n_{l} \rightarrow \infty$ and $x_{k_{l}}^{n_{l}} \rightarrow x$, then $x \in F$. Since $v_{n} \rightarrow u_{1}$ and $x_{k}^{n} \rightarrow v_{n}$ as $k \rightarrow-\infty$, for each $\epsilon>0$, one can find a sequence $x_{k_{l}}^{n_{l}}$ as above and satisfying $\epsilon / 2 \leq\left\|u_{1}-x_{k_{l}}^{n_{l}}\right\| \leq \epsilon$. As this sequence belongs to the compact set $J$, we conclude that there exist points of $F$ arbitrarily close to $u_{1}$. Let $y_{n} \in F \backslash\left\{u_{1}\right\}$ and $y_{n} \rightarrow u_{1}$. Obviously, $S_{t}\left(y_{n}\right)>y_{n}$ ( $y_{n}$ is not an equilibrium) and $S_{t}\left(y_{n}\right) \rightarrow u_{2}$ as $t \rightarrow \infty$. Now $u_{1}$ does not belong to the compact set $F \cap\left[e, u_{2}\right]$ so there exists $r>0$ such that $\bar{B}\left(r, u_{1}\right)$ has no point in common with this set. For each $n$, there exists $t_{n}>0$ such that $\left\|S_{t_{n}}\left(y_{n}\right)-u_{1}\right\|=r$ and $t_{n} \rightarrow \infty$ since $y_{n} \rightarrow u_{1}$. It follows that there is a subsequential limit $z$ of $\left\{S_{t_{n}}\left(y_{n}\right)\right\}$ which belongs to $\omega(F)$ and satisfies $\left\|z-u_{1}\right\|=r$. As $\omega(F)$ is an invariant subset of $F$, there exists an entire orbit $\gamma: \mathbb{R} \rightarrow \omega(F)$ of $S$ through $z$ (see [3]). Because the entire orbit belongs to $F$, it is monotone and since $z$ is not an equilibrium, the orbit is strictly monotone. Its limits as $t \rightarrow \pm \infty$ are equilibria and therefore its limit as $t \rightarrow+\infty$ must be $u_{2}$. Since $y$ does not belong to $F \cap\left[e, u_{2}\right]$, it follows that $\gamma(t) \rightarrow u_{1}$ as $t \rightarrow-\infty$. This completes our sketch.

If $Y^{+}$has nonempty interior in $Y, u_{1} \ll u_{2}$, and whenever $u \in I$ is an equilibrium of $S$ distinct from $u_{1}, u_{2}, e$, it follows that $u_{1} \ll u \ll u_{2}$, then precisely one of alternatives (a)-(c) of Proposition 2.4 holds. The proof mirrors the proof in the discrete case.

\section{Proof of MAin Results}

In this section, we use the notation developed in section 1 . We begin by considering the restriction of $T$ to the set

$$
I \equiv\left[E_{2}, E_{1}\right]_{K} \equiv\left\{x \in X: E_{2} \leq_{K} x \leq_{K} E_{1}\right\}=\left[0, \hat{x}_{1}\right] \times\left[0, \tilde{x}_{2}\right]
$$

Note that $I$ is an order interval with respect to the ordering $\leq_{K}$. Our first result is an immediate consequence of Proposition 2.1.

Theorem 3.1. Let $T$ satisfy (H1), (H2) and (H3). Then $T(I) \subset I$ and at least one of the following holds:

(a) $T$ has a fixed point distinct from $E_{0}, E_{1}, E_{2}$ in $I$.

(b) There is an entire orbit $\left\{x_{n}\right\}_{n \in Z}$ of $T$ in $I$ joining $E_{2}$ to $E_{1}$ and satisfying $x_{n}<_{K} x_{n+1}$. 
(c) There is an entire orbit $\left\{x_{n}\right\}_{n \in Z}$ of $T$ in $I$ joining $E_{1}$ to $E_{2}$ and satisfying $x_{n+1}<_{K} x_{n}$.

Proof. We apply Proposition 2.1 to $T$ on the order interval $I \subset X^{+}$. By strict monotonicity of $T$ with respect to $<_{K}$, we conclude that $T(I) \subset I$. Observe that $E_{0}$ is an extreme point of $I$ and, by (H2), it is an ejective fixed point of $T$. Since $T$ is order compact, it follows that $T(I)$ has compact closure in $I$. Therefore, the hypotheses of Proposition 2.1 are satisfied and Theorem 3.1 follows from it.

As observed in the previous section, the inequality $E_{2} \ll_{K} E_{1}$ implies that alternatives (b) and (c) cannot both hold. Next we show that if (H4) is satisfied, then exactly one of (a)-(c) holds.

Proposition 3.2. Let $T$ satisfy (H1)-(H4). Then precisely one of alternatives (a)-(c) of Theorem 3.1 hold. Furthermore, if $T$ has a fixed point $E_{*}$ in I distinct from $E_{0}, E_{1}, E_{2}$, then $E_{2} \ll_{K} E_{*} \ll_{K} E_{1}$ and, in particular, $0 \ll E_{*}$. If (b) holds, then

$$
E_{2} \ll_{K} x_{n} \ll_{K} x_{n+1} \ll_{K} E_{1},
$$

while if (c) holds, then

$$
E_{2} \ll_{K} x_{n+1} \ll_{K} x_{n} \ll_{K} E_{1},
$$

for all $n \in Z$.

Proof. If $E_{*}=\left(x_{1}, x_{2}\right)$ is a fixed point distinct from $E_{0}, E_{1}, E_{2}$, then $x_{i} \neq 0$ for $i=1,2$ by $(\mathrm{H} 3)$. By (H4), $0 \ll E_{*}=T\left(E_{*}\right)$ and by (H4), we may conclude that $E_{2} \ll_{K} E_{*} \ll_{K} E_{1}$. Proposition 2.2 implies that precisely one of the alternatives (a)-(c) holds. Suppose now that (b) holds. If $x_{n}=\left(x_{1}, x_{2}\right)$, then $x_{i} \neq 0$ for $i=1,2$ by (H3). As $n$ is arbitrary and $x_{n}=T\left(x_{n-1}\right)$, we conclude that $0 \ll x_{n}$. Since $E_{2}<_{K} x_{n-1}<_{K} E_{1}$, it follows from (H4) that $E_{2} \ll_{K} x_{n} \ll_{K} E_{1}$. Similarly, $x_{n-1}<_{K} x_{n}$ implies $x_{n} \ll_{K} x_{n+1}$ by (H4).

Lemma 3.3. Let $T$ satisfy (H1)-(H4). If alternative (b) of Theorem 3.1 holds, then $T^{n}(x) \rightarrow E_{1}$ for all $x=\left(x_{1}, x_{2}\right) \in I$ such that $x_{i} \neq 0, i=1,2$. If alternative (c) holds, then $T^{n}(x) \rightarrow E_{2}$ for all such $x$.

Proof. Suppose (b) holds. By (H4), $T(x) \gg 0$. As $E_{2}<_{K} T(x)<_{K} E_{1}$, (H4) implies that $E_{2} \ll_{K} T^{2}(x) \ll_{K} E_{1}$. We may choose $n \in Z$ such that $E_{2} \ll_{K}$ $x_{n} \ll_{K} T^{2}(x)$. By monotonicity, it follows that $x_{n+l} \ll_{K} T^{l+2}(x) \ll_{K} E_{1}$ for $l=1,2, \ldots$ Letting $l \rightarrow \infty$ and noting that $O(x)$ has compact closure leads to the desired conclusion since $x_{m} \rightarrow E_{1}$.

Proof of Theorem A. In order to prove Theorem A, we must extend Lemma 3.3 by removing the restriction that $x \in I$. We begin by showing that $I$ attracts all orbits. According to (H4), we may as well assume from the start that $0 \ll T^{n}(x)$ for all $n$. If $x=\left(x_{1}, x_{2}\right)$, let $u=\left(x_{1}, 0\right)$ and $v=\left(0, x_{2}\right)$ and observe that $v<_{K} x<_{K} u$. Consequently,

$$
T^{n}(v)<_{K} T^{n}(x)<_{K} T^{n}(u)
$$

for all $n \geq 1$. (H1) implies that $T^{n}(v) \rightarrow E_{2}$ and $T^{n}(u) \rightarrow E_{1}$. In particular, if $s>1$, then $T^{n}(x) \in\left[0, s \hat{x}_{1}\right] \times\left[0, s \tilde{x}_{2}\right]$ for all large $n$. As $T$ is order compact, we conclude that $O(x)$ has compact closure in $X$. It follows that the omega limit set is nonempty, compact, invariant $(T(\omega(x))=\omega(x))$, and invariantly connected 
[11]. The latter means that $\omega(x)$ is not the disjoint union of two nonempty, closed, invariant subsets. From the inequality above, $\omega(x) \subset I$.

Suppose that alternative (b) of Proposition 3.2 holds. Let $z \in \omega(x)$ and $z=$ $\left(z_{1}, z_{2}\right)$. If $z_{i} \neq 0$ for $i=1,2$, then $T(z) \gg 0$ and therefore $E_{2} \ll_{K} T^{2}(z) \ll_{K} E_{1}$ by (H4). In this case, since $T^{2}(z) \in \omega(x)$, there exists $m$ such that $E_{2} \ll_{K} T^{m}(x) \ll_{K}$ $E_{1}$. But then $T^{n}(x) \rightarrow E_{1}$ as desired. Therefore, we may now assume that for every $z \in \omega(x), z_{i}=0$ for exactly one index $i$, either $i=1$ or $i=2(0 \notin \omega(x)$ or else we are in the previous case). Now $\omega(x)$ is invariantly connected so by (H3), either $z_{1}=0$ for all $z \in \omega(x)$ or $z_{2}=0$ for all $z \in \omega(x)$. By (H3), the only compact invariant subset of $X_{1}^{+} \times\{0\}$ which does not include $(0,0)$ is $E_{1}$. A symmetric conclusion holds for the other 'axis' $\{0\} \times X_{2}^{+}$. We conclude that either $\omega(x)=\left\{E_{2}\right\}$ or $\omega(x)=\left\{E_{1}\right\}$.

Proof of Corollary 1. The existence of a positive fixed point of $T$ in case (i) holds follows immediately from Theorem A since alternative (b) ((c)) of the theorem implies that $E_{2}\left(E_{1}\right)$ is unstable relative to $I$ in the order topology (or the usual topology). If case (iii) holds, then, by Theorem $\mathrm{A}, z \in I$ and it is obvious from Theorem A that neither alternative (b) nor (c) can hold. Finally, if alternative (b) of Theorem A holds, then by Proposition 3.2, there is an entire orbit $\left\{x_{n}\right\}_{n \in Z}$ satisfying $E_{2} \ll_{K} x_{n} \ll_{K} x_{n+1} \ll_{K} E_{1}$ and $x_{n} \rightarrow E_{1}$ as $n \rightarrow \infty$. This implies that $E_{1}$ is stable (actually asymptotically stable) relative to $I$ in the order topology. In fact, to show the stability relative to $I$ in the order topology, it suffices to show that given $x^{*} \in X$ satisfying $x^{*} \ll_{K} E_{1}$, there exists $y^{*} \in X$ with $y^{*} \ll_{K} E_{1}$ such that if $y^{*} \ll_{K} x \leq_{K} E_{1}$, then $x^{*} \ll_{K} T^{n}(x) \leq_{K} E_{1}$ for $n \geq 1$. As $x_{n} \rightarrow E_{1}$, there exists $x_{n}$ such that $x^{*} \ll_{K} x_{n} \ll_{K} E_{1}$. If $x_{n} \ll_{K} x \leq_{K} E_{1}$, then $x^{*} \ll_{K} x_{n} \ll_{K} x_{n+l}<_{K}$ $T^{l}(x) \leq_{K} E_{1}$ for $l \geq 1$ so $y^{*}$ can be taken to be $x_{n}$ and $E_{1}$ is stable relative to $I$ in the order topology. This contradicts (ii). Similarly, alternative (c) of Theorem A implies that $E_{2}$ is stable relative to $I$ in the order topology, contradicting (ii). Thus alternative (a) must hold if (ii) holds.

Proof of Proposition 2. Let $x_{*}$ denote a positive fixed point of $T$, which by (H4) must satisfy $E_{2} \ll_{K} x_{*} \ll_{K} E_{1}$. The set $W=\left\{\bar{x}: T(\bar{x})=\bar{x}, x_{*} \leq_{K} \bar{x} \ll_{K} E_{1}\right\}$ is partially ordered by $\leq_{K}$. Using the compactness of the set of fixed points, the fact that $E_{1}$ is an isolated fixed point, and Lemma 2.3 one can show that $W$ has a maximal element which we call $E_{*}$. Obviously, the order interval $\left[E_{*}, E_{1}\right]_{K}$ contains no fixed points of $T$ other than $E_{*}$ and $E_{1}$. Now we use the result of Dancer and Hess [2], our Proposition 2.1 but without the ejective fixed point $e$, to conclude that there exists a monotone entire orbit $\left\{x_{n}\right\}$ which either connects $E_{*}$ to $E_{1}$ or connects $E_{1}$ to $E_{*}$. Suppose the former holds. Then, arguing as in the proof of Proposition 3.2, we have $E_{*} \ll_{K} x_{n} \ll_{K} x_{n+1} \ll_{K} E_{1}$ for all $n \geq 1$. If $x=\left(x_{1}, x_{2}\right)$ satisfies $E_{*}<_{K} x \leq_{K} E_{1}$ and $x_{2} \neq 0$, then $E_{*}<_{K} T(x)<_{K} E_{1}$ and $T(x)$ is positive so $E_{*} \ll_{K} T^{2}(x)<_{K} E_{1}$. We can choose $x_{n}$ so that $x_{n} \ll_{K} T^{2}(x)$ and it follows by monotonicity that $T^{n}(x) \rightarrow E_{1}$. The same conclusion holds if $x_{2}=0$ by (H3). A similar argument shows that (i) holds if $\left\{x_{n}\right\}$ connects $E_{1}$ to $E_{*}$.

It should be pointed out that the preceding proofs can be easily modified to allow a slight weakening of (H4). If $x=\left(x_{1}, x_{2}\right)$ satisfies $x_{i} \neq 0$, then it suffices to assume that there exists an integer $m$ such that $0 \ll T^{m}(x)$. In the continuous version, we could assume that for each such $x$, there exists $t_{0}>0$ such that $T_{t_{0}}(x) \gg 0$. 
The proof of Theorem B is obtained from Proposition 2.4 by straightforward extensions of the results of section 3 to the case of semiflows.

\section{REFERENCES}

1. H. Amann, Fixed point equations and nonlinear eigenvalue problems in ordered Banach spaces, SIAM Rev. 18 (1976), 620-709. MR 57:7269

2. E. Dancer and P. Hess, Stability of fixed points for order-preserving discrete-time dynamical systems, J. Reine Angew. Math. 419 (1991), 125-139. MR 92i:47088

3. J.K. Hale, Asymptotic Behavior of Dissipative Systems, AMS Math. Surv. and Monogr., vol. 25, 1988. MR 89g:58059

4. J.K. Hale and P. Waltman, Persistence in infinite dimensional systems, SIAM J. Math. Anal. 20 (1989), 388-395. MR 90b:58156

5. M. Hirsch, Stability and convergence in strongly monotone dynamical systems, J. Reine Angew. Math. 383 (1988), 1-53. MR 89c:58108

6. P. Hess, Periodic-parabolic Boundary Value Problems and Positivity, Longman Scientific and Technical, New York, 1991. MR 92h:35001

7. P. Hess and A.C. Lazer, On an abstract competition model and applications, Nonlinear Analysis T.M.A. 16 (1991), 917-940. MR 92f:92036

8. S.B. Hsu and P. Waltman, On a system of reaction-diffusion equations arising from competition in an unstirred chemostat, SIAM J. Appl. Math. 53 (1993), 1026-1044. MR 95a:92015

9. S.B. Hsu, P. Waltman, and S. Ellermeyer, A remark on the global asymptotic stability of a dynamical system modeling two species in competition, Hiroshima J. Math. 24 (1994), 435446. MR 95h:35115

10. S.B. Hsu, H.L. Smith, and P. Waltman, Dynamics of Competition in the unstirred chemostat, Canadian Applied Math. Quart. 2 (1994), 461-483. MR 96f:92011

11. J.P. LaSalle, The Stability of Dynamical Systems, SIAM, 1976. MR 58:1426

12. R. Nussbaum, Periodic Solutions of some nonlinear autonomous functional differential equations, Ann. Mat. Pura. Appl. 101 (1974), 263-306. MR 50:13817

13. _ The Fixed Point Index and Some Applications, University of Montreal Press, 1985. MR 87a: 47085

14. P. Polacik, Convergence in smooth strongly monotone flows defined by semilinear parabolic equations, J. Diff. Eqns. 79 (1989), 89-110. MR 90f:58025

15. H.L. Smith, B. Tang, and P. Waltman, Competition in an n-vessel chemostat, SIAM J. Appl. Math. 5 (1991), 1451-1471. MR 92m:92016

16. H.L. Smith, Monotone Dynamical Systems, An Introduction to the Theory of Competitive and Cooperative Systems, AMS Math. Surv. and Monogr., vol. 41, 1995. MR 96c:34002

17. H.L. Smith and H. Thieme, Convergence for strongly ordered preserving semiflows, SIAM J. Math. Anal. 22 (1991), 1081-1101. MR 92m:34145

18. H.L. Smith and P. Waltman, The Theory of the Chemostat, Cambridge University Press, 1995. MR 96e:92016

19. P. Takáč, Discrete monotone dynamics and time-periodic competition between two species, preprint.

20. J. Wu, H.I. Freedman, and R.K. Miller, Heteroclinic orbits and convergence of orderpreserving set-condensing semiflows with applications to integrodifferential equations, J. Integral Equations and Applications 7 (1995), 115-133. MR 96c:34165

Institute of Applied Mathematics, National Tsing Hua University, Hsinchu, Taiwan E-mail address: sbhsu@am.nthu.edu.tw

Department of Mathematics, Arizona State University, Tempe, Arizona 85287-1804

E-mail address: halsmith@math.la.asu.edu

Department of Mathematics, Emory University, Atlanta, Georgia 30322

E-mail address: waltman@mathcs.emory.edu 